\title{
ON THE TREATMENT OF OBSTRUCTIVE DYSMENORRHCEA AND STERILITY.
}

\author{
Br THOMAS MORE MADDEN, M.D., F.R.C.S.E.; \\ Obstetric Physician, Mater Misericordiæ Hospital, Dublin ; \\ President, Obstetric Section, British Medical Association.
}

[Read in the Section of Obstetrics, March 16, 1888.]

The treatment of sterility and obstructive dysmenorrhoea aro closely interwoven questions of great practical interest, which, up to the present time, have not been referred to in the Transactions of the Royal Academy of Medicine. Hence, although I have elsewhere considered certain aspects of these subjects, I now venture to submit my views thereon, some of which $I$ believe will be found novel to this Academy, in the hope of inducing a discussion on one of the most frequent, and of tentimes the most difficult, of the daily-recurring problems that come under gynæcological attention.

In so doing I would premise that it appears to me in the recognition of obstruction, from either cervical or fallopian stenosis, as the chief cause of sterility as well as of dysmenorrhœa, will be found the key to the pathology and successful treatment of the great majority of cases of both these morbid conditions. These, as already observed, being intimately connected in their pathology and treatment, must be here dealt with in conjunction, although with special reference to the former. The frequency of such cases is much greater than is generally admitted, as in my hospital practice, during the past eleven years, upwards of eight hundred instances of obstructive dysmenorrhoea, or of sterility similarly caused, have come under observation in a total of seven thousand gynæcological cases that have been there noted in our intern and extern departments. Nor is the pathological importance of these complaints less striking than their frequency. Thus, of all the ailments of female existence, few give rise to greater and more 
persistent suffering, or produce more disastrous effects not only on the general physical health, but also on the cerebro-nervous system, and on the moral constitution of the patient, than result from wellmarked obstructive dysmenorrhœa. The importance of the lastmentioned consequence of dysmenorrhœa, to which I directed special attention in an article of mine on the Disorders of the Female Sexual Functions, in Quain's Dictionary of Medicine, is by no means as generally recognised as it should be. This is more especially evident in many cases of alcoholism, and I may repeat that a morbid craving for alcohol in women may very frequently be dated from their first painful menstrual period, when stimulants are too often forced by foolish mothers into the generally reluctant lips of their daughters. Once, however, the pain of dysmenorrhœa has been thus relieved, the girl at the next epoch of suffering naturally, and no longer unwillingly, seeks similar solace, until finally the victim of dysmenorrhceal alcoholism becomes an habitual, and, perhaps, an incurable drunkard.

Importance of the Study of Sterility.-The effects of the second of the morbid conditions that form the subject of this communication are certainly of no less moment, in a practical aspect, than are those results to which $I$ have just referred as consequent on the first-named ailment-the latter involving not only the physical health and well-being of our patients, but also, in too many instances, intimately affecting their social interests, and the happiness of conjugal life. In this country, at least, the infamous doctrines of the modern votaries of "Venus sine Lucilla" have, fortunately to no appreciable extent, displaced the Christian view of the main function and chief end of woman's married life. Hence, in Ireland to the present time, as in Israel in the days of Sarah, to be childless is commonly regarded as a reproach to a married woman, and as a subject of marital disappointment and trouble.

Causes of Barrenness.-As in every other morbid condition so in the treatment of sterility-our first aim must be to ascertain, and our second to remove, if it be removable, the cause of the trouble for which we are consulted. Of the various causes of barrenness, some, 
as for instance the absence or arrested development of the organs essential for conception-viz., the uterus, fallopian tubes, or ovariæ, being beyond remedial reach, need not here occupy our consideration. In the great majority of instances, however, sterility, occurring in women within the limits of ovarian functional vitality, admits of effectual treatment, when that treatment is rationally directed to the special exigencies of each case.

By most authorities on this subject, including even the late Dr. Marion Sims, female sterility, or reproductive inability, has been confounded with impotency or sexual incapacity. Hence, it may not appear superfluous to remind my hearers that, properly speaking, the latter term should be restricted to those cases in which marital intercourse is prevented by some physical impediment or malformation in the female genital tract-such as vaginal or vulval occlusion, imperforate hymen, vaginismus, cervical stenosis, \&c., by which impregnation may be precluded, whilst sterility or imperfection of conceptive power is due to either some structural lesion, deficiency, displacement, or flexion of some one of the organs essential to conception. Thus, the uterus, fallopian tubes, or ovariæ, may each be absent, misplaced, or otherwise abnormal, or else these parts, being in a normal condition, the patient nevertheless remains sterile, either from constitutional causes or from other causes-such as sexual irrespondence or incongruity, of a moral rather than of a physical nature.

Stenosis of the Cervical Canal.-This, according to my experience, now extending over a quarter of a century, in hospital and in private consultation, at home and abroad, is not only the most frequent of all the causes of sterility, but is, moreover, the most amenable to appropriate treatment of all the physical factors in the causation of infecundity. Until a recent period, however, neither the frequency nor the rational treatment of this cause of barrenness were recognised by modern surgeons, although both were clearly pointed out by some of the older writers. Thus it was not until the late Sir James Simpson recalled attention to the plan of dilating the os uteri and cervical canal by a method which, several years previously, had been suggested by another Edinburgh surgeon, whose 
ignored suggestion on the use of sponge tents in the treatment of dysmenorrhœa Simpson revived, that anything like adequate attention was attracted to this important subject. But both Simpson and Mackintosh were forestalled in their views by ancient writers, whose names had long been forgotten, and whose works had apparently become hermetically sealed by the hand of time. Hence, as I believe that the history of the steps by which modern gynæcological science has arrived at its present development are well worthy of the consideration of all who pride themselves on being members of a learned and liberal profession, $I$ venture, in this connection, to recapitulate, briefly, a few observations which I made several years since, before a society which no longer exists, on this point.

The credit of suggesting the dilatation of the os uteri and cervical canal by means of sponge tents is, as already said, generally conceded to the late Sir James Simpson, by whom it was claimed, in a paper of his published in the Edinburgh Journal of Medical Science for January, 18j0, in which he says-"In 1844, in a communication laid before the Medico-Chirurgical Society of Ediuburgh, I proposed a means of safely opening up the cavity of the cervix and body of the uterus to such an extent as might enable us to introduce a finger into the uterine cavity for the purpose of diagnosis in the treatment of this (uterine polypi) and other morbid conditions of the organ. The means described consisted in the introduction of sponge tents into the os and cavity of the uterus, so as gradually to dilate these parts to the degree required." This suggestion was then looked on as "marking the commencement of a new era in uterine surgery." It is, therefore, not a little interesting to find that a similar method of dilating the os uteri was well known and described some two centuries and a half ago. Thus, in "The Method of Physic, containing the causes, signs, and cures of inward diseases in man's body from the head to the foot," by Philip Barrough, and "most humbly dedicated by the author to his singular good lord and master, Lord Burghley," the eighth edition of which was published in 1639 , the writer, speaking of the treatment of contraction of the cervical canal and os uteri giving rise 
to mechanical dysmenorrhœa, and producing sterility, \&c., in the chapter entitled "Of Straightness of the Matrice," says:- "And when the places do seem to be softer to the feeling, then you must put a dry sponge, that hath a cord hanged at it, into the straight place, to the intent to make it wider, which, if it fall out, you must put in another that is thicker. Therefore, you must have many and sundry dry sponges ready." This ancient gynæcologist next refers to the possibility of these sponge tents producing inflammation in the mouth or neck of the matrice, and discusses its treatment, after which he continues-"When the inflammation is ceased, and the place is open, annoint upon a sponge a cerot made of oill of roses and goose grease, and use it untill it be healed, making the place a little sounder; but yet you must alwaies put in sponges untill the end of the cure, lest that the mouth of the womb do gather together againe."

This coincidence does not detract from the merit of Sir James Simpson, to whom suffering humanity, as well as medical science, owes so much on other seores, as one of the first modern gynæcologists to make a practical application of tents in the treatment of a condition then commonly regarded as beyond the reach of curative treatment. I may, however, take this opportunity of observing that the number of such coincidences between long-forgotten ideas and so-called modern medical discoveries is far greater than could be readily credited by those who share in that illiterate neglect of the lessons to be gathered from the experience of the past, as embodied in the works of the older medical writers, and in that ignorant contempt for their opinions and observations which is, unfortunately, too prevalent at the present day.

It is not my purpose here to refer in detail to the successive improvements which have since been effected in the methods of carrying out the gradual dilatation of the cervical canal, the most inportant of which were due to the late Dr. Sloan, who introduced the use of laminaria tents as a substitute for the old-fashioned and now, happily, generally disused fotid and inefficient sponge tents ; and to Dr. Kidd, who improved on Dr. Sloan's idea by the employment of a number of laminaria bougies to secure a com- 
paratively more rapid and complete expansion. Fortunately, we have now passed beyond the period of gynæcological practice in which these more slow, painful, and often hazardous plans of cervical dilatation were the only methods available. No greater improvement has occurred in our branch of surgery than the replacement of these of tentimes unsatisfactory procedures by the more effective means now at our disposal for the rapid expansion of this canal. Of these, perhaps, the best known and most generally employed in this country are either Hegar's or Lawson Tait's dilators. I now desire to call your attention to another instrument which $I$ have designed for the same purpose, and which, I venture to hope, may be found by others, as it has by myself, more satisfactory in its use in the treatment of dysmenorrhoea and sterility occasioned by stenosis.

This instrument will, I believe, be found to supply a want long recognised by gynæcologists-namely, that of a reliable and effective means of securing the rapid and permanent dilatation of the cervical canal in the treatment of stenosis giving rise to the morbid conditions now under consideration. The principles on which it is constructed differ from that of other dilators in several respects, and, above all, in one which I consider most important-viz., in producing expansion of the canal from within outwards-in other words, in imitating the natural process of expansion from the uterine cavity downwards to the os uteri; whereas other dilators,

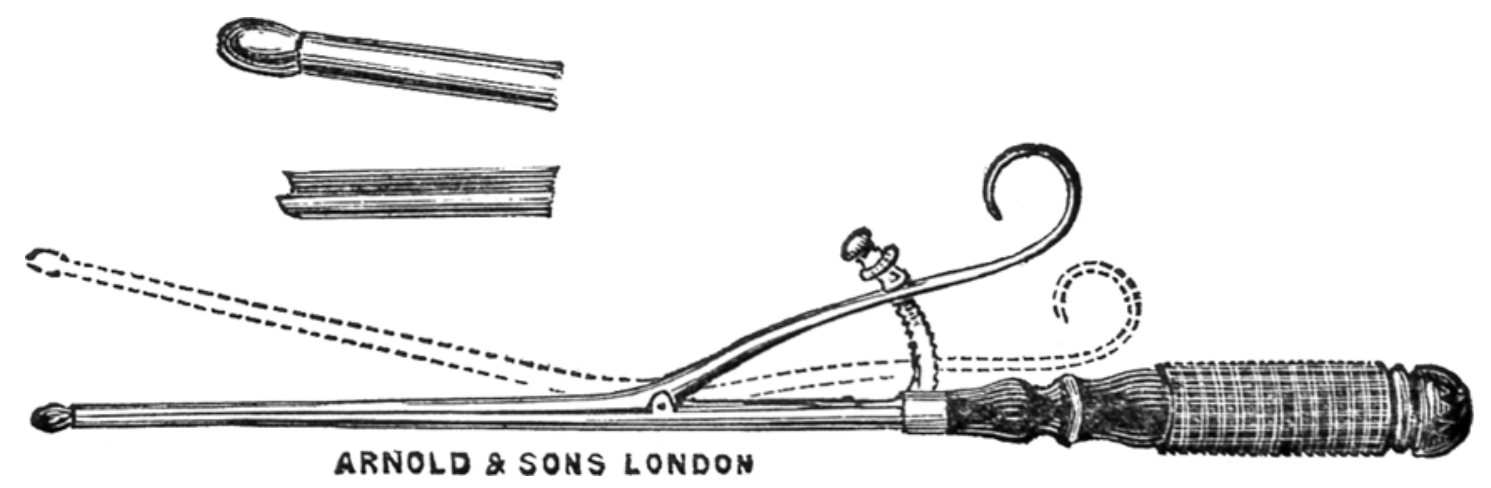

such as Hegar's, \&c., act in the opposite direction. In my own hands the utility of this instrument, the expansion effected by which may be determined and accurately measured by the affixed index, has been fully tested in a large number of cases of sterility and dysmenorrhoea in hospital and private practice. I may add that the instrument, which does not occupy more room than the ordinary 
sound when introduced, may also be used with advantage for the dilatation of the female urethra in many cases in which this procedure is indicated. This instrument has been carefully made in accordance with my directions by Messrs. Arnold and Sons, West Smithfield, London.

Operative Treatment of Cervical Stenosis.-As the method I employ in these cases differs in some respects from that generally adopted, I may be here permitted to refer briefly to its details. I may observe that during the past eleven years $I$ have operated on several hundred cases of stenosis, and within the last year I thus successfully treated in my hospital no less than ninety-eight such cases, in many of which $I$ had the able assistance of my friend, Dr. Duke, Obstetric Physician to Steevens' Hospital.

In the first place, I would advise that this operation should be undertaken about a week after the termination of the last painful menstrual period, and that it should be preceded by daily hot water syringing for some days. To proceed with any comfort to ourselves or advantage to our patient, she should be placed under ether or mythelene, and adjusted in the ordinary left lateral semiprone position, on a proper gynæcological couch or table. Then, the cervix being exposed by the duck-bill speculum; the anterior lip is seized by a strong vulsellum, and drawn down by an assistant as close to the vulva as possible, where it is to be retained. Next the sound should be passed to ascertain the position of the uterus, as well as the immediate permeability of the canal. Then the metrotome (and I myself much prefer Simpson's original instrument to any of its modifications) should be introduced well into the uterine cavity, and, being expanded to whatever extent may be deemed necessary in each case, should be withdrawn so as to divide the cervix freely in a transverse direction from the os internum to the outer orifice of the passage. It should be again introduced, and the same procedure repeated downwards, and again in an antero-posterior direction. The uterine cavity should now be syringed out with hot water (temp. $100^{\circ}$ ), so as to wash away all clots and arrest any hæmorrhage, then $I$ introduce the dilator already described, and, expanding this to its full extent, 
I allow it to remain for a few moments, and then withdraw the instrument, with blades apart, so as to tear asunder and distend the cut surfaces until I can pass my index in to the uterine cavity. In this way any probability of either hæmorrhage or of subsequent re-union of the divided parts is effectually provided against. Lastly, $I$ introduce a tampon of Lawton's cotton saturated in glycerine of carbolic acid, which is left in situ for some days so as to fill and distend the passage from the uterine cavity to the os externum. The introduction of this may be found by no means an easy matter by the uninitiated, and can best be effected by the employment of the sliding repositor.

After the operation the patient must be kept in bed on low diet, and syringed daily with hot water for eight or ten days, any pain being meanwhile treated by opiates and poultices. After this period a flexible tube or uterine stem pessary is introduced, which should be worn for another month, and then, and not sooner, the patient may resume marital life with a fair probability of subsequent impregnation, and almost absolute certainty of immunity from any recurrence of dysmenorrhœa.

With regard to the results of this operation, my experience has been most satisfactory in the curative treatment of a large proportion of the cases of sterility and obstructive dysmenorrhœea that have come under my observation. This result I ascribe mainly to the thoroughness with which I carry out the dilatation and disruption of the contracted passage in the cases referred to. And as I have been reproached by some more timid practitioners with what they regard as the rashness or boldness of my practice in this respect, I may here venture to add that when cautiously, albeit boldly, carried out, in appropriate cases and by expert hands, no ill results need attend the operation in question, and I myself have had no such reason to regret its performance. In only two cases have I seen any serious hæmorrhage after it. Nor have I found it necessary to plug the vagina in such cases, as I have seen done by some practitioners, to the great discomfort of the patient. In only as many instances has any inflammatory symptoms or cellulitis followed its performance, and in both these cases the operation had been performed by other gynæcologists previously. 
Besides cervical stenosis there are other causes of obstructive dysmenorrhcea and sterility which must also be considered in this connection. Of these, perhaps the most frequent and most important is vaginismus. As, however, I have discussed the pathology and treatment of this condition in a previous paper, I need here only briefly refer to those points which bear most directly on the subject of the present communication.

Excessive sensibility of the vaginal orifice and adjacent parts, more especially when associated with such spasmodic contraction of the sphincter vaginæ as to form an impediment to marital intercourse, or dyspareunia, occasionally becomes a matter of considerable gynæcological interest in connection with the treatment of sterility. In such cases the hyperæsthetic condition of the vaginal outlet is evinced even on the slightest attempt at local examination, and is most marked about the meatus urinarius, and in the vicinity of the orifice of the vulvo-vaginal glands and fourchette, whence the hymen, if existent, projects upwards.

With regard to the pathology of vaginismus, there are almost as many divergent views as there have been writers on the subject. For my own part $I$ think the most rational explanation of these symptoms may generally be found in the hysterical temperament of the majority of those thus affected, although in some cases there is also present an abnormal condition of the pudic nerve, one branch of which runs along with the artery to the clitoris, whilst the other, or superficial perinæal nerve, is distributed to the perinæum and labia, in which its terminal branches ratify freely. This fact in the rtiology of the disease is one the practical importance of which will be seen in connection with the treatment of raginismus.

Treatment.-It was long since said by Dr. Marion Sims that there is " no disease capable of producing so much unhappiness to both parties of the marriage contract." I regret to say that in my own experience in some instances I have found no little difficulty in dealing with extreme cases of this kind by any of the plans of treatment generally recommended. As a rule, in the treatment of such cases very undue importance is given to local operative measures, whilst 
the constitutional treatment which is invariably necessary in all instances of well-marked vaginismus is too generally neglected. For whilst excision of the hymen, division of the pudic nerve, destruction of erythematous and serpiginous patches, dissections out of neuromata, \&c., may each be indispensable in certain cases, in quite as many they are unnecessary. And, from my own clinical experience, $I$ can vouch for the possibility in some cases of relieving the most intense dyspareunia resulting from this cause, so as to enable the patient to fulbl all her duties as a wife and eventually as a mother, without any operation beyond the forcible mechanical expansion of the vaginal canal. Before resorting even to this expedient we should, in the first place, employ the sedative treatment, local and general, which is indicated in all other nervous or hysterial disorders, and which, as I believe, is essential in nine-tenths of the cases of vaginismus that come before us, whilst in only one-tenth of them is any surgical or operative measure necessary.

Amongst the topical palliative remedies that may, conjointly with the constitutional nerve sedatives just referred to, be employed in these cases, are warm baths and vaginal irrigations, local application of solution of hydrochlorate of cocain or of glycerine of carbolic acid, or the introduction of suppositories of cocain and belladonna. When such palliative measures have been fairly tried without advantage, we may then resort to mechanical dilatation of the vaginal orifice and stretching of the pudic nerve. For this purpose, having first fully etherised the patient, a large-sized Graily-Hewitt bivalve speculum should be introduced and expanded to its fullest extent. Then a tampon of absorbent lotion large enough to fill the speculum should be soaked in glycerine and passed up to the cervix, its lower end projecting through the external opening of the instrument. This, still fully expanded, should then be forcibly drawn out, leaving the central tampon behind in the vagina. It need hardly be observed that this procedure occasions severe pain. At the same time, however, it as certainly tears through some of the superficial submucous muscular fibres of the affected part, as well as effec- 
tually stretches the terminal vaginal branches of the pudic nerve, and thus affords a generally efficient and safe method of overcoming the spasmodic contraction with which we have to deal in cases of vaginismus. Any subsequent contraction or hæmorrhage that may follow this procedure is sufficiently met by the tampon, which may be retained for at least twenty-four hours; and after some days, should there be still a continuance of vaginismus, the same method of treatment may be again repeated.

In some cases, however, this method of treatment does not suffice, and we must then resort to either Emmet's or to Sims's operation for vaginismus. In some instances $I$ have found the hæmorrhage following these operations sufficiently serious, and in one of these I had to thank my friend Dr. Horne, our Hon. Secretary, who was called in in my absence, for the arrest of very alarming loss of blood, occurring some hours after the operation, in the case of a young lady on whom I performed Sims's operation.

It should here be observed that even in cases of vaginismus so extreme as to effectually prevent complete marital intercourse, the disease is not necessarily an absolute barrier to impregnation. In one instance of this kind that came under my observation some years ago, so extreme was the local hyperæsthesia as not only to preclude the probability of complete cohabitation, but also to prevent the patient's submitting to any local treatment for the relief of the morbid condition. Nevertheless conception occurred, and I subsequently was called in to deliver her at full term, and in doing so was obliged to incise the still unruptured hymen, by which delivery was obstructed.

Obstructive Dysmenorrhcea and Sterility from Vaginal Occlusion.Amongst the causes of the morbid conditions under consideration some reference must be here made to occlusion of the vaginal passage whether by congenital malformations, or, as more frequently met with in gynæcological practice, from cicatricial adhesions the result of disease or accident. With regard to the latter, although I have met with them in several instances, our clinical experience of retained menstruation, dispareunia, and impeded delivery 
consequent on post-partum adhesions of the vaginal walls, is fortunately more limited than must have been the case in the practice of our professional predecessors, in those good old days when the second stage of labour was allowed to run on until the vital powers were all but completely exhausted, before instrumental assistance was afforded in cases of difficult parturition. Nevertheless, such cases are still occasionally met with.

Of somewhat greater frequency in the causation of this condition in modern practice is the opposite error. In other words, the integrity of the vaginal canal is now more likely to be injured by the application of the forceps, before the sufficient natural dilatation of the passage, or by the misdirected force or undue haste with which instrumental delivery may be effected by injudicious practitioners.

Another occasional source of cicatricial obstruction or obliteration of the vagina is the abuse of escharotics or instruments intended for the treatment of uterine disease. It may also possibly result from syphititic disease as well as from phagedænic ulceration or direct injuries to the vulvo-vaginal orifice.

In the first volume of the Transactions of this Academy I have referred to cases of vaginal occlusion, and I regret that time prevents my now dwelling further on this subject, as such cases appear to me of much interest, not only from their comparative infrequency, but still more from the possibility of this condition being an occasional cause of menstrual retention and an obstacle to impregnation or parturition. Moreover, they would serve to illustrate the special risk attending the surgical treatment of cicatricial vaginal obstructions. The latter point is one which should never be lost sight of in such cases. It was demonstrated by a distinguished Irish obstetrician, the late Dr. Evory Kennedy, in The Dublin Quarterly Journal many years ago, that the treatment of vaginal occlusion is by no means as safe as it is facile. This observation has been corroborated by more recent writers, and amongst others by MM. Bernutz and Goupil, who have shown conclusively the risk of dividing these obstructions or adhesions by any cutting instrument, and the comparative safety of digital 
separation-the mortality following the use of the knife as compared with the surgeon's finger in such cases being almost as three to one.

Chronic Endo-cervicitis as a Cause of Sterility.-Lastly, we must here refer to another and much more frequent factor in the causation of obstructive sterility and dysmenorrhœa, namely, what was formerly termed chronic inflammation, and since more properly designated areolar hyperplasia, of the cervix uteri. A brief glance at the pathology of this condition will show its importance in relation to the study of sterility. Suffice it to say that the theory by which this disease was for many years ascribed to idiopathic inflammation, leading to ulceration of the cervix uteri, has been long since abandoned, except by its author, and that it is now as generally regarded as the result of passive congestion, or hyperæmia, commencing in the cervical mucous membrane, the ciliated epithelium of which is thus primarily tumefied and softened, whilst later on the whole tract of the endo-uterine mucous membrane becomes disintegrated and hypertrophied. After a little time this hyperplasia extends to the subjacent muscular structures of the uterus generally, and gives rise therein to those sero-plastic exudations and infiltrations by which the affected parts are at first mechanically distended and thickened. As the disease goes on these plastic exudations becoming organised eventuate in new connective tissue, or true congestive hypertrophy, or areolar hyperplasia of the uterus. At the same time follicular degeneration of the hypertrophied cervical glands occurs, and in their subsequent proliferation in most instances may be found the explanation of the conditions formerly regarded as the result of ulceration of the cervix uteri. The structural differences between the endo-uterine and endocervical mucous membrane are also of direct interest in this connection, explaining to some extent the greater proneness of the latter to kyperæmia, and the greater probability of this condition in the former being more immediately communicated to the underlying parenchyma. For whilst the ciliate membrane lining the cavity of the uterus is directly superimposed on the muscular substance, without the intervention of any sub-mucous layer, and 
has numerous utricular glands, separated merely by prolongations of the uterine muscular fibre, the endo-cervical mucous membrane, on the contrary, rests on a sub-mucous layer, and is arranged in numerous folds branching off on either side from the central ridge, and thus presenting the appearance known as the arbor-vita. This membrane is lined with ciliated epithelium, and interposed between its folds are countless racemose glands or Nabothian follicles opening between the ridges of the arbor-vitæ, and under diseased eonditions pouring out that viscid, white-of-egg-like secretion so generally found blocking the os uteri and cervical canal in gynæeological examinations.

In such cases the functions of the uterus are invariably disturbed. The menses are abnormal; generally they are attended by severe pain; in some instances they are diminished, but more commonly, menorrhagia as well as dysmenorrhoea results from the local congestion and irritation; and in a large proportion of cases of this kind I have observed that the abnormal menstruation recurred every third week, and lasted for six or seven days, whilst in the interval the patient was further weakened by the leucorrhœal diseharge already spoken of.

Sterility almost always accompanies this disease, and as long as it exists to any serious extent the patient must remain barren. This fact, which I regard as of great practical importance, is too generally ignored in practice. I have known instances in which patients were subjected to active surgieal treatment to overcome a supposed mechanical obstacle to impregnation, and who nevertheless remained ehildless, no attention having been paid to the existence of chronic uterine congestion, on the subsequent cure of which pregnancy has followed.

Ovarian and tubal inflammation, manifested by soreness, tumefaction, and occasionally burning pain in the ovarian region, is one of the most frequent consequences and accompaniments of uterine hyperæmia. In these cases the disease extends from the uterus, along the Fallopian tubes to the ovaries, and this to a great extent accounts for the fact $I$ have just mentioned, that patients suffering from endo-metritis or endo-cervicitis, are sterile for the time being. 
Were it not that $I$ have already possibly exceeded the prescribed limits of time here allowed to readers of papers, I should have wished to state my experience with regard to the treatment of cases of sterility and obstructive dysmenorrhoea due, as is so of ten the ease, to uterine flexions and displacements, or to fallopian tubal or ovarian causes, to which latter very great importance should be assigned in this connection, as well as to those just referred to. I must, however, rest content, for the present at least-as I hope to be allowed to return to this important subject on some future occasion-with having, in the foregoing communication, described the method which $I$ have found most successful in a large proportion of the numerous cases of barrenness and painful menstruation which have come under my care in the gynæcological department of my hospital and in private practice during the last few years. Moreover, I have also pointed out more briefly the principles which I believe should guide our treatment in the other forms of the complaints alluded to. I trust that the Academy will pardon the length at which $I$ have thus trespassed on their attention, and by the free discussion of the views I have now submitted, aid other practitioners in the curative treatment of a class of cases second to none in their importance and frequency. 